\title{
ON THE DIAGONALIZATION OF THE RICCI FLOW ON LIE GROUPS
}

\author{
JORGE LAURET AND CYNTHIA WILL
}

(Communicated by Lei $\mathrm{Ni}$ )

\begin{abstract}
The main purpose of this note is to prove that any basis of a nilpotent Lie algebra for which all diagonal left-invariant metrics have diagonal Ricci tensor necessarily produce quite a simple set of structural constants; namely, the bracket of any pair of elements of the basis must be a multiple of one of them, and only the bracket of disjoint pairs can be a nonzero multiple of the same element. Some applications to the Ricci flow of left-invariant metrics on Lie groups concerning diagonalization are also given.
\end{abstract}

\section{INTRODUCTION}

The classification of all possible signatures for the Ricci curvature of left-invariant metrics on 3-dimensional unimodular Lie groups obtained by Milnor in [M76] relies on the following key fact: any such Lie algebra admits a basis such that the corresponding diagonal metrics represent all left-invariant metrics up to isometry and, moreover, their Ricci tensors are all diagonal as well. This of course has also been crucial in the study of the Ricci flow and Ricci solitons for these metrics in IJ92, KM01, G08, CS09, GP10, which actually cover most 3-dimensional geometries from the Geometrization Conjecture. Indeed, if a basis $\left\{X_{1}, \ldots, X_{n}\right\}$ of a Lie algebra is stably Ricci-diagonal in the sense that any diagonal left-invariant metric (i.e. $\left\langle X_{i}, X_{j}\right\rangle=0$ for all $i \neq j$ ) has diagonal Ricci tensor, then the set of diagonal metrics is invariant under the Ricci flow and hence the qualitative study of the solutions reduces considerably. These distinguished bases were introduced and named by Payne in $\mathrm{P} 10$, where a deep study of the qualitative behavior of the Ricci flow for those left-invariant metrics on nilpotent Lie groups admitting such a basis has been done.

It is therefore natural to ask which Lie algebras admit a stably Ricci-diagonal basis or, even further, which left-invariant metrics admit an orthonormal stably Ricci-diagonal basis. For instance, the study in [IJL06] of the Ricci flow on homogeneous 4-manifolds could not be carried out for the totality of left-invariant metrics (even on the 3-step nilpotent Lie group) due to the lack of these special bases (see also [Lt07]).

From a more algebraic point of view, there is a condition on the basis of a Lie algebra based on the simplicity of the corresponding set of structural constants. Namely, a basis $\left\{X_{1}, \ldots, X_{n}\right\}$ of a Lie algebra is said to be nice if $\left[X_{i}, X_{j}\right]$ is

Received by the editors November 1, 2011 and, in revised form, December 30, 2011.

2010 Mathematics Subject Classification. Primary 53C30, 53C44.

This research was partially supported by grants from CONICET (Argentina) and SeCyT (Universidad Nacional de Córdoba). 
always a scalar multiple of some element in the basis and two different brackets $\left[X_{i}, X_{j}\right],\left[X_{r}, X_{s}\right]$ can be a nonzero multiple of the same $X_{k}$ only if $\{i, j\}$ and $\{r, s\}$ are disjoint. As far as we know, this concept first appeared in the literature in [LW11, Lemma 3.9] and has been widely used in the study of nilsolitons (i.e. Ricci soliton nilmanifolds). For instance, Nikolayevsky obtained a very useful and simple criterium to decide whether a given nilpotent Lie algebra with a nice basis admits a nilsoliton or not (see [N11, Theorem 3]). An updated overview of the existence of these bases in the nilpotent case is given in Section 2 .

Let $(N, g)$ be a nilpotent Lie group endowed with a left-invariant metric (called a nilmanifold for short). If $(\mathfrak{n},\langle\cdot, \cdot\rangle)$ is the corresponding metric Lie algebra (i.e. the value $g(e)$ of the metric on the tangent space at the identity element $\mathrm{T}_{e} N=\mathfrak{n}$ is $\langle\cdot, \cdot\rangle)$, then the Ricci tensor is given by

(1) $\operatorname{Rc}(X, Y)=-\frac{1}{2} \sum\left\langle\left[X, X_{i}\right], X_{j}\right\rangle\left\langle\left[Y, X_{i}\right], X_{j}\right\rangle+\frac{1}{4} \sum\left\langle\left[X_{i}, X_{j}\right], X\right\rangle\left\langle\left[X_{i}, X_{j}\right], Y\right\rangle$,

for any $X, Y \in \mathfrak{n}$ and any orthonormal basis $\left\{X_{1}, \ldots, X_{n}\right\}$ of $(\mathfrak{n},\langle\cdot, \cdot\rangle)$.

It follows at once from (11) that any nice basis is stably Ricci-diagonal. Indeed, the basis $\left\{X_{1}, \ldots, X_{n}\right\}$ is orthogonal relative to any diagonal metric $\langle\cdot, \cdot\rangle$, and, clearly, each summand in the formula for $\operatorname{Rc}\left(X_{r}, X_{s}\right)$ vanishes when $r \neq s$. The main object of this note is to prove that the converse assertion also holds.

Theorem 1.1. A basis of a nilpotent Lie algebra is stably Ricci-diagonal if and only if it is nice.

A purely geometric condition on a basis of a nilpotent Lie algebra is therefore characterized as a neat algebraic condition. The proof is based on the fact that the Ricci operator is precisely the moment map for the $\mathrm{GL}_{n}(\mathbb{R})$-representation where the Lie brackets live. In the nonnilpotent case, none of the implications in the theorem remain true (see Examples [5.4, 5.5, 5.6).

Back to the Ricci flow, what Theorem 1.1 is saying is that, in the case of a nilmanifold $\left(N, g_{0}\right)$, to ask for a stably Ricci-diagonal basis in order to get a diagonal Ricci flow solution $g(t)$ starting at $g_{0}$ is quite expensive (see [L10] for a study of the Ricci flow on nilmanifolds without the use of these special bases). We prove for instance that any algebraic soliton $\left(N, g_{0}\right)$ (i.e. $\operatorname{Ric}\left(g_{0}\right) \in \mathbb{R} I \oplus \operatorname{Der}(\mathfrak{n})$, a condition which easily implies that $\left(N, g_{0}\right)$ is indeed a Ricci soliton), where $N$ is any (nonnecessarily nilpotent) Lie group, gives rise to a diagonal Ricci flow solution (see Example 5.2).

On the other hand, already in dimension 4, there are nilmanifolds whose Ricci flow is not diagonal with respect to any orthonormal basis (see Example 5.7).

\section{ON THE EXISTENCE OF A NICE BASIS}

Let $\mathfrak{n}$ be a nilpotent Lie algebra. A basis $\left\{X_{1}, \ldots, X_{n}\right\}$ of $\mathfrak{n}$ is called nice if the structural constants given by $\left[X_{i}, X_{j}\right]=\sum c_{i j}^{k} X_{k}$ satisfy

- for all $i, j$ there exists at most one $k$ such that $c_{i j}^{k} \neq 0$,

- for all $i, k$ there exists at most one $j$ such that $c_{i j}^{k} \neq 0$.

$\mathfrak{n}$ is said to be of type $\left(n_{1}, \ldots, n_{r}\right)$ if $n_{i}=\operatorname{dim} C^{i-1}(\mathfrak{n}) / C^{i}(\mathfrak{n})$, where $C^{i}(\mathfrak{n})$ is the central descendent series of $\mathfrak{n}$ defined by $C^{0}(\mathfrak{n})=\mathfrak{n}, C^{i}(\mathfrak{n})=\left[\mathfrak{n}, C^{i-1}(\mathfrak{n})\right]$. One can always take a direct sum decomposition $\mathfrak{n}=\mathfrak{n}_{1} \oplus \ldots \oplus \mathfrak{n}_{r}$ such that $C^{i}(\mathfrak{n})=$ $\mathfrak{n}_{i+1} \oplus \ldots \oplus \mathfrak{n}_{r}$ for all $i$, and so $\operatorname{dim} \mathfrak{n}_{i}=n_{i}$. 
If the type of $\mathfrak{n}$ is $\left(n_{1}, \ldots, n_{r}\right)$, then we can reorder any nice basis so that the first $n_{1}$ elements give a basis for $\mathfrak{n}_{1}$, the following $n_{2}$ give a basis for $\mathfrak{n}_{2}$, and so on. This easily follows from the fact that a nonzero bracket $\left[X_{i}, X_{j}\right]$ must be a multiple of some $X_{k}$.

Concerning existence, from the explicit bases exhibited in the classification lists available in the literature, we have that any nilpotent Lie algebra of dimension $\leq 5$ as well as any filiform $\mathbb{N}$-graded Lie algebra admits a nice basis (see e.g. [N08b]). Also, by using the classification of 6-dimensional nilpotent Lie algebras given for example in dG07, it is easy to check that all of the 34 algebras in the list are written in a nice basis with the exception only of $L_{6,11}$, which is denoted by $\mu_{11}$ in W03. We now prove that indeed this algebra cannot have a nice basis (this fact has independently been mentioned in F11 after Definition 2.7, including an idea of the proof).

Proposition 2.1. The 6-dimensional 4-step nilpotent Lie algebra of type $(3,1,1,1)$ defined by

$$
\left[X_{1}, X_{2}\right]=X_{4}, \quad\left[X_{1}, X_{4}\right]=X_{5}, \quad\left[X_{1}, X_{5}\right]=\left[X_{2}, X_{3}\right]=\left[X_{2}, X_{4}\right]=X_{6},
$$

does not admit any nice basis.

Proof. Let $\mathfrak{n}$ be a 4 -step nilpotent Lie algebra of type $(3,1,1,1)$ with no abelian factor. Assume that $\left\{X_{1}, \ldots, X_{6}\right\}$ is a nice basis of $\mathfrak{n}$ so that $\mathfrak{n}_{1}=\operatorname{span}\left\{X_{1}, X_{2}, X_{3}\right\}$, $\mathfrak{n}_{2}=\mathbb{R} X_{4}, \mathfrak{n}_{3}=\mathbb{R} X_{5}$ and $\mathfrak{n}_{4}=\mathbb{R} X_{6}$. Thus $X_{6}$ is in the center of $\mathfrak{n}$ and there exist $i, j, k, l \in\{1,2,3\}$ such that

$$
\left[X_{i}, X_{j}\right]=a X_{4}, \quad\left[X_{k}, X_{4}\right]=b X_{5}, \quad\left[X_{l}, X_{5}\right]=c X_{6}, \quad a, b, c \neq 0 .
$$

Note that since the basis is nice, the brackets $\left[X_{1}, X_{2}\right],\left[X_{1}, X_{3}\right],\left[X_{2}, X_{3}\right]$, if nonzero, are linearly independent. We therefore have three cases to consider:

$$
d:=\operatorname{dim}\left\langle\left[X_{1}, X_{2}\right],\left[X_{1}, X_{3}\right],\left[X_{2}, X_{3}\right]\right\rangle=1,2 \text { or } 3,
$$

and in any case we can assume that $\left[X_{1}, X_{2}\right]=X_{4}$.

The simplest one corresponds to $d=1$, where by using Jacobi identities it is easy to see that $\mathbb{R} X_{3}$ is an abelian factor. If $d=3$, that is,

$$
\left[X_{1}, X_{2}\right]=X_{4}, \quad\left[X_{1}, X_{3}\right]=X_{5}, \quad\left[X_{2}, X_{3}\right]=X_{6},
$$

then we also must have

$$
\left[X_{2}, X_{4}\right]=b^{\prime} X_{5}, \quad\left[X_{1}, X_{5}\right]=c^{\prime} X_{6},
$$

for some $b^{\prime}, c^{\prime} \in \mathbb{R}$. The Jacobi identities now imply that $b^{\prime} c^{\prime}=0$, and therefore (3) would not hold.

Finally, if $d=2$, we may assume that

$$
\left[X_{1}, X_{2}\right]=X_{4}, \quad\left[X_{2}, X_{3}\right]=\alpha X_{5}+\beta X_{6},
$$

where either $\alpha \neq 0$ or $\beta \neq 0$. If $\beta=0$, by assuming that $\alpha=1$, we obtain that $k=1$ and $\left[X_{3}, X_{4}\right]=-\left[X_{1}, X_{5}\right]$. Thus the nontrivial Lie brackets are

$$
\begin{array}{rrr}
{\left[X_{1}, X_{2}\right]=X_{4},} & {\left[X_{2}, X_{3}\right]=X_{5},} & {\left[X_{3}, X_{4}\right]=X_{6},} \\
{\left[X_{1}, X_{4}\right]=b X_{5},} & {\left[X_{1}, X_{5}\right]=-X_{6},}
\end{array}
$$

for some $b \neq 0$. A change of bases given by

$$
\left\{X_{1}, X_{2}, \epsilon X_{3}, \epsilon^{-1} X_{4}, \epsilon X_{5}, \epsilon X_{6}\right\},
$$


where $\epsilon=|b|^{1 / 2}$, shows that $\mathfrak{n}$ is isomorphic to $L_{6,13}$. A similar argument shows that if $\alpha=0$, we get $L_{6,12}$.

We have then showed that any type- $(3,1,1,1) 4$-step nilpotent Lie algebra with no abelian factor that admits a nice basis must be isomorphic to either $L_{6,12}$ or $L_{6,13}$, and thus $L_{6,11}$ cannot admit a nice basis. We note that the fact that these three algebras are pairwise nonisomorphic also follows by using the fact that they admit nilsolitons of different eigenvalue types (compare with $\mu_{9}, \mu_{10}$ and $\mu_{11}$ in W03, Tables 1, 3]).

A distinguished class of nilpotent Lie algebras admitting a nice basis is given by nilradicals of Borel subalgebras of any semisimple Lie algebra.

On the other hand, it is proved in [N11, Example 4] that the free 3-step nilpotent Lie algebra in 3 generators (which is of type $(3,3,8)$ ) does not admit a nice basis, and, by a dimensional argument, it is also shown that there exist infinitely many 2-step nilpotent Lie algebras with no nice basis for any type $(p, q)$ such that

$$
\frac{1}{2} \min \{q(q-1), p q\}+q^{2}+p^{2}-1<\frac{1}{2} p q(q-1) .
$$

This condition holds, for example, for any $q-1 \geq p \geq 6$, and the 13-dimensional case $(6,7)$ is the lowest one. In dimension 7 , by using the classification given in Mg07, one can check that from the 117 algebras and 6 curves of 7 -dimensional indecomposable nilpotent Lie algebras, all but 27 algebras and 2 curves are written in a nice basis (see [F11]). We do not know whether these exceptions admit a nice basis or not.

We can also mention the following sufficient condition: any nilpotent Lie algebra admitting a simple derivation (i.e. diagonalizable over $\mathbb{R}$ and with all its eigenvalues of multiplicity one) has a nice basis. Indeed, any basis of eigenvectors $\left\{X_{1}, \ldots, X_{n}\right\}$ of $D$ is automatically nice since $\left[X_{i}, X_{j}\right]$ is either 0 or also an eigenvector of $D$, and if $\left[X_{i}, X_{j}\right],\left[X_{r}, X_{s}\right] \in \mathbb{R} X_{k}$, then the (pairwise different) eigenvalues satisfy $d_{i}+d_{j}=d_{r}+d_{s}=d_{k}$. This implies that $\{i, j\}$ and $\{r, s\}$ are either equal or disjoint. We note that this is not a necessary condition. One may check, for example in [W11, Tables 4-6], that the 6-dimensional algebras $\mu_{8}$ and $\mu_{18}$ do not admit any simple derivation and that they both admit a nice basis.

\section{TECHNICAL PRELIMINARIES}

Let us consider the space of all skew-symmetric algebras of dimension $n$, which is parameterized by the vector space

$$
V=\Lambda^{2}\left(\mathbb{R}^{n}\right)^{*} \otimes \mathbb{R}^{n}=\left\{\mu: \mathbb{R}^{n} \times \mathbb{R}^{n} \longrightarrow \mathbb{R}^{n}: \mu \text { bilinear and skew-symmetric }\right\} .
$$

There is a natural linear action of $\mathrm{GL}_{n}(\mathbb{R})$ on $V$ defined by

$$
A \cdot \mu(X, Y)=A \mu\left(A^{-1} X, A^{-1} Y\right), \quad X, Y \in \mathbb{R}^{n}, \quad A \in \mathrm{GL}_{n}(\mathbb{R}), \quad \mu \in V,
$$

and the corresponding representation of the Lie algebra $\mathfrak{g l}_{n}(\mathbb{R})$ of $\mathrm{GL}_{n}(\mathbb{R})$ on $V$ is given by

$$
\pi(\alpha) \mu=\alpha \mu(\cdot, \cdot)-\mu(\alpha \cdot, \cdot)-\mu(\cdot, \alpha \cdot), \quad \alpha \in \mathfrak{g l}_{n}(\mathbb{R}), \quad \mu \in V .
$$

The canonical inner product $\langle\cdot, \cdot\rangle$ on $\mathbb{R}^{n}$ determines inner products on $V$ and $\mathfrak{g l}_{n}(\mathbb{R})$, both also denoted by $\langle\cdot, \cdot\rangle$, as follows:

$$
\langle\mu, \lambda\rangle=\sum_{i j k}\left\langle\mu\left(e_{i}, e_{j}\right), e_{k}\right\rangle\left\langle\lambda\left(e_{i}, e_{j}\right), e_{k}\right\rangle, \quad\langle\alpha, \beta\rangle=\operatorname{tr} \alpha \beta^{\mathrm{t}},
$$


where $\left\{e_{1}, \ldots, e_{n}\right\}$ denotes the canonical basis of $\mathbb{R}^{n}$ and $\beta^{t}$ the transpose with respect to $\langle\cdot, \cdot\rangle$. We note that $\pi(\alpha)^{t}=\pi\left(\alpha^{t}\right)$ and $(\operatorname{ad} \alpha)^{t}=\operatorname{ad} \alpha^{t}$ for any $\alpha \in \mathfrak{g l}_{n}(\mathbb{R})$, due to the choice of canonical inner products everywhere.

We use $\mathfrak{g l}_{n}(\mathbb{R})=\mathfrak{s o}(n) \oplus \operatorname{sym}(n)$ as a Cartan decomposition of $\mathfrak{g l}_{n}(\mathbb{R})$, where $\mathfrak{s o}(n)$ and $\operatorname{sym}(n)$ denote the subspaces of skew-symmetric and symmetric matrices, respectively. The set $\mathfrak{a}$ of all diagonal $n \times n$ matrices is a maximal abelian subalgebra of $\operatorname{sym}(n)$ and therefore determines a system of roots $\Delta \subset \mathfrak{a}$. Let $\Phi$ denote the set of positive roots, which is given by

$$
\Phi=\left\{E_{l l}-E_{m m} \in \mathfrak{a}, \quad l>m\right\},
$$

where $E_{r s}$ denotes the matrix whose only nonzero coefficient is 1 at entry $r s$. We have the root space decomposition $\mathfrak{g l}_{n}(\mathbb{R})=\mathfrak{a} \oplus \underset{\lambda \in \Delta}{\bigoplus} \mathfrak{g}_{\lambda}$, where for each $\lambda \in \Delta$,

$$
\mathfrak{g}_{\lambda}=\left\{X \in \mathfrak{g l}_{n}(\mathbb{R}):[\alpha, X]=\langle\alpha, \lambda\rangle X, \quad \forall \alpha \in \mathfrak{a}\right\} .
$$

If $\left\{e_{1}^{\prime}, \ldots, e_{n}^{\prime}\right\}$ is the basis of $\left(\mathbb{R}^{n}\right)^{*}$ dual to the canonical basis $\left\{e_{1}, \ldots, e_{n}\right\}$, then

$$
\left\{v_{i j k}=\left(e_{i}^{\prime} \wedge e_{j}^{\prime}\right) \otimes e_{k}: 1 \leq i<j \leq n, 1 \leq k \leq n\right\}
$$

is a basis of weight vectors of $V$ for the representation (5), where $v_{i j k}$ is actually the bilinear form on $\mathbb{R}^{n}$ defined by $v_{i j k}\left(e_{i}, e_{j}\right)=-v_{i j k}\left(e_{j}, e_{i}\right)=e_{k}$ and zero otherwise. The corresponding weights $\alpha_{i j}^{k} \in \mathfrak{a}, i<j$, are given by

(8) $\pi(\alpha) v_{i j k}=\left(a_{k}-a_{i}-a_{j}\right) v_{i j k}=\left\langle\alpha, \alpha_{i j}^{k}\right\rangle v_{i j k}, \quad \forall \alpha=\left[\begin{array}{ccc}a_{1} & & \\ & \ddots & \\ & & a_{n}\end{array}\right] \in \mathfrak{a}$, where $\alpha_{i j}^{k}:=E_{k k}-E_{i i}-E_{j j}$.

The representation $(V, \pi)$ of $\mathfrak{g l}_{n}(\mathbb{R})$ is multiplicity free; i.e. the weight spaces all have dimension one. An additional special feature of this representation is that the sum of two weights $\alpha_{i j}^{k}+\alpha_{r s}^{t}$ is never zero.

\section{Proof of Theorem 1.1}

By fixing any basis of an $n$-dimensional nilpotent Lie algebra $\mathfrak{n}$, we can identify its underlying vector space $\mathfrak{n}$ with $\mathbb{R}^{n}$ and view its bracket $[\cdot, \cdot]$ as an element of $V=\Lambda^{2}\left(\mathbb{R}^{n}\right)^{*} \otimes \mathbb{R}^{n}$ (see Section 3 for all definitions and notation used in what follows). The structural constants $c_{i j}^{k}$ are therefore given by

$$
\left[e_{i}, e_{j}\right]=\sum_{k} c_{i j}^{k} e_{k} \quad \text { or } \quad[\cdot, \cdot]=\sum_{k ; i<j} c_{i j}^{k} v_{i j k} .
$$

We note that

$\mathfrak{n}$ admits a nice basis if and only if the canonical basis $\left\{e_{1}, \ldots, e_{n}\right\}$

is nice for some $A \cdot[\cdot, \cdot] \in V$ with $A \in \mathrm{GL}_{n}(\mathbb{R})$,

as this is precisely the 'change of basis' action. Let us first show that the nice condition on a bracket $[\cdot, \cdot]$, in the sense that the canonical basis is nice for $\mathfrak{n}$, can be written in terms of the set $\Phi$ of positive roots of $\mathfrak{g l}_{n}(\mathbb{R})$ (see (17)) and the weights of the representation $(V, \pi)$ (see (8) ) .

Lemma 4.1. The canonical basis $\left\{e_{1}, \ldots, e_{n}\right\}$ is nice for $\mathfrak{n}$ if and only if

$$
\alpha_{i j}^{k}-\alpha_{r s}^{t} \notin \Phi, \quad \text { for any } \quad c_{i j}^{k}, c_{r s}^{t} \neq 0 .
$$


Proof. According to (2), $\left\{e_{1}, \ldots, e_{n}\right\}$ is nice for $\mathfrak{n}$ if and only if

$$
\sharp\left\{k: c_{i j}^{k} \neq 0\right\} \leq 1 \quad \text { and } \quad \sharp\left\{j: c_{i j}^{k} \neq 0\right\} \leq 1 .
$$

If $\alpha_{i j}^{k}-\alpha_{r s}^{t} \in \Phi$ for some $c_{i j}^{k}, c_{r s}^{t} \neq 0$, then

$$
E_{k k}-E_{i i}-E_{j j}-E_{t t}+E_{r r}+E_{s s}=E_{l l}-E_{m m},
$$

for some $l>m$. Since $\mathfrak{n}$ is nilpotent, we have that $i, j \neq k$ as soon as $c_{i j}^{k} \neq 0$ under any of the two conditions in the lemma. Indeed, if $c_{i j}^{i} \neq 0$, then there must exist $k$ such that $c_{i j}^{k} \neq 0$, and so $\left\{e_{1}, \ldots, e_{n}\right\}$ would not be nice and $\alpha_{i j}^{i}-\alpha_{i j}^{k}$ would equal $E_{k k}-E_{i i} \in \pm \Phi$. It is therefore easy to check that the cancellation given in (10) can only be possible when either $\{i, j\}=\{r, s\}$ and $t<k$ or $t=k$ and $\sharp(\{i, j\} \cap\{r, s\})=1$. In any case, one of the conditions in (9) will not hold. In other words, $\alpha_{i j}^{k}-\alpha_{r s}^{t} \in \Phi$ for some $c_{i j}^{k}, c_{r s}^{t} \neq 0$ if and only if $\left\{e_{1}, \ldots, e_{n}\right\}$ is not a nice basis for $\mathfrak{n}$, as was to be shown.

We note that each $\mu \in V$ satisfying the Jacobi identity determines a Lie group $N_{\mu}$ (the simply connected Lie group with Lie algebra $\left(\mathbb{R}^{n}, \mu\right)$ ), which can be endowed with the left-invariant metric defined by the canonical inner product $\langle\cdot, \cdot\rangle$ (fixed). Let us denote by $\mathrm{Rc}_{\mu}$ the Ricci tensor of such a metric and by $\mathrm{Ric}_{\mu}$ its Ricci operator. The action of $\mathrm{GL}_{n}(\mathbb{R})$ on $V$ given by (4) has the following geometric interpretation: each $A \in \mathrm{GL}_{n}(\mathbb{R})$ determines a Riemannian isometry

$$
\left(N_{A \cdot \mu},\langle\cdot, \cdot\rangle\right) \longrightarrow\left(N_{\mu},\langle A \cdot, A \cdot\rangle\right)
$$

by exponentiating the Lie algebra isomorphism $A^{-1}:\left(\mathbb{R}^{n}, A \cdot \mu\right) \longrightarrow\left(\mathbb{R}^{n}, \mu\right)$. It follows from (11) that

the canonical basis $\left\{e_{1}, \ldots, e_{n}\right\}$ is stably Ricci-diagonal for $\mathfrak{n}$ if and only if the matrix of $\operatorname{Ric}_{A \cdot[\cdot, \cdot]}$ is diagonal for any diagonal $A \in$ $\mathrm{GL}_{n}(\mathbb{R})$.

It is proved in [L06, Proposition 3.5] that when $\mu$ is in addition nilpotent,

$$
\left\langle\operatorname{Ric}_{\mu}, \alpha\right\rangle=\frac{1}{4}\langle\pi(\alpha) \mu, \mu\rangle, \quad \forall \alpha \in \operatorname{sym}(n) .
$$

Remark 4.2. This is equivalent to saying that the moment map $m: V \backslash\{0\} \longrightarrow$ $\operatorname{sym}(n)$ for the action (44) is given by $m(\mu)=\frac{4}{\|\mu\|^{2}} \operatorname{Ric}_{\mu}$, a remarkable fact which is really at the core not only of the main result obtained in the present paper but actually of the whole subject of Ricci flow and solitons on nilmanifolds (see [L09, L10]).

In light of what has already been shown in this section, Theorem 1.1 follows from the equivalence between parts (i) and (iv) in the following theorem.

Theorem 4.3. For a nilpotent Lie algebra $\mathfrak{n}$, the following conditions are equivalent:

(i) The canonical basis $\left\{e_{1}, \ldots, e_{n}\right\}$ is nice for $\mathfrak{n}$.

(ii) $\left\langle\pi(X) v_{i j k}, v_{r s t}\right\rangle=0$, for all $X \in \mathfrak{g}_{\lambda}, \lambda \in \Phi, c_{i j}^{k}, c_{r s}^{t} \neq 0$.

(iii) $\operatorname{Rc}_{A \cdot \mu}\left(e_{l}, e_{m}\right)=0$ for all $l \neq m$ and any diagonal $A \in \mathrm{GL}_{n}(\mathbb{R})$.

(iv) The canonical basis $\left\{e_{1}, \ldots, e_{n}\right\}$ is stably Ricci-diagonal for $\mathfrak{n}$.

Proof. We first note that parts (iii) and (iv) are equivalent by (11), as was mentioned above. By evaluating the maps $\pi([\alpha, X])=\pi(\alpha) \pi(X)-\pi(X) \pi(\alpha)$ at $v_{i j k}$ and then taking scalar product with $v_{r s t}$, we obtain

$\left\langle\lambda+\alpha_{i j}^{k}, \alpha\right\rangle\left\langle\pi(X) v_{i j k}, v_{r s t}\right\rangle=\left\langle\alpha_{r s}^{t}, \alpha\right\rangle\left\langle\pi(X) v_{i j k}, v_{r s t}\right\rangle, \quad \forall X \in \mathfrak{g}_{\lambda}, \quad \lambda \in \Phi, \quad \alpha \in \mathfrak{a}$, 
from which it follows that

$$
\left\langle\pi(X) v_{i j k}, v_{r s t}\right\rangle \neq 0 \quad \Longrightarrow \quad \alpha_{r s}^{t}-\alpha_{i j}^{k}=\lambda \in \Phi .
$$

This implies that part (ii) follows from (i) and Lemma 4.1

Conversely, assume that part (ii) holds. If $\left\{e_{i}\right\}$ is not nice, by Lemma 4.1 there exist $c_{i j}^{k}, c_{r s}^{t} \neq 0$ such that $\alpha_{r s}^{t}-\alpha_{i j}^{k}=E_{l l}-E_{m m} \in \Phi$. By using

$$
\pi\left(E_{l m}\right) v_{i j k}=\delta_{k m} v_{i j l}-\delta_{i l} v_{m j k}-\delta_{j l} v_{i m k},
$$

it is easy to check that $\left\langle\pi\left(E_{l m}\right) v_{i j k}, v_{r s t}\right\rangle \neq 0$ if $i, j, k$ are pairwise different. Otherwise, if say $j=k$ (and hence $t=r$ or $t=s$ ), then by arguing as in the proof of Lemma 4.1, we obtain that there exists $k^{\prime} \neq i, j$ such that $c_{i j}^{k^{\prime}} \neq 0$ and $\left\langle\pi\left(E_{k^{\prime} i}\right) v_{i j k^{\prime}}, v_{i j k}\right\rangle \neq 0$. Part (i) therefore follows since for all $l>m, E_{l m} \in \mathfrak{g}_{\lambda}$ for $\lambda=E_{l l}-E_{m m} \in \Phi$.

By using (12) and the fact that for any $A=\operatorname{Diag}\left(a_{1}, \ldots, a_{n}\right) \in \mathrm{GL}_{n}(\mathbb{R}), A \cdot v_{i j k}=$ $\frac{a_{k}}{a_{i} a_{j}} v_{i j k}$, we get that

$$
\begin{aligned}
4 \operatorname{Rc}_{A \cdot[\cdot, \cdot]}\left(e_{l}, e_{m}\right) & =\left\langle\pi\left(E_{l m}\right) A \cdot[\cdot, \cdot], A \cdot[\cdot, \cdot]\right\rangle \\
& =\sum c_{i j}^{k} c_{r s}^{t}\left\langle\pi\left(E_{l m}\right) A \cdot v_{i j k}, A . v_{r s t}\right\rangle \\
& =\sum c_{i j}^{k} c_{r s}^{t} \frac{a_{k}}{a_{i} a_{j}} \frac{a_{t}}{a_{r} a_{s}}\left\langle\pi\left(E_{l m}\right) v_{i j k}, v_{r s t}\right\rangle,
\end{aligned}
$$

and thus part (iii) follows from (ii).

Finally, we will show that part (iii) implies (ii). We begin by noting that if $A=\exp (\alpha), \alpha \in \mathfrak{a}$, then

$$
\exp (\alpha) \cdot v_{i j k}=e^{\left\langle\alpha_{i j}^{k}, \alpha\right\rangle} v_{i j k} .
$$

It follows as in (15) and by using part (iii) that for all $l>m$,

$$
0=4 \operatorname{Rc}_{A \cdot[\cdot, \cdot]}\left(e_{l}, e_{m}\right)=\sum c_{i j}^{k} c_{r s}^{t} e^{\left\langle\alpha_{i j}^{k}+\alpha_{r s}^{t}, \alpha\right\rangle}\left\langle\pi\left(E_{l m}\right) v_{i j k}, v_{r s t}\right\rangle,
$$

where the sum runs only over the indices $i j k$ and $r s t$ such that $\alpha_{i j}^{k}-\alpha_{r s}^{t}=$ $E_{l l}-E_{m m} \in \Phi$, since otherwise $\left\langle\pi\left(E_{l m}\right) v_{i j k}, v_{r s t}\right\rangle=0$ by (13). Also, notice that $\left\langle\pi\left(E_{l m}\right) v_{i l i}, v_{r m r}\right\rangle=0$ for all $i, r$ (see (14) $)$, and so we can assume from now on that $i, j, k$ and $r, s, t$ are respectively pairwise different.

If $\alpha_{i_{o} j_{o}}^{k_{o}}-\alpha_{r_{o} s_{o}}^{t_{o}}=E_{l l}-E_{m m}$, then there exists $\alpha_{o} \in \mathfrak{a}$ such that $0 \neq\left\langle\alpha_{i_{o} j_{o}}^{k_{o}}+\right.$ $\left.\alpha_{r_{o} s_{o}}^{t_{o}}, \alpha_{o}\right\rangle$ and $\left\langle\alpha_{i_{o} j_{o}}^{k_{o}}+\alpha_{r_{o} s_{o}}^{t_{o}}, \alpha_{o}\right\rangle \neq\left\langle\alpha_{i j}^{k}+\alpha_{r s}^{t}, \alpha_{o}\right\rangle$ for any pair ijk, rst of indices with $\alpha_{i j}^{k}-\alpha_{r s}^{t}=E_{l l}-E_{m m}$ (note that $\alpha_{i_{o} j_{o}}^{k_{o}}+\alpha_{r_{o} s_{o}}^{t_{o}} \neq \alpha_{i j}^{k}+\alpha_{r s}^{t}$ for any such 3-uple of indices). It follows from (16) that

$$
0=\sum c_{i j}^{k} c_{r s}^{t}\left\langle\pi\left(E_{l m}\right) v_{i j k}, v_{r s t}\right\rangle e^{t\left\langle\alpha_{i j}^{k}+\alpha_{r s}^{t}, \alpha_{o}\right\rangle}=\sum_{n=1}^{N} a_{n} e^{t b_{n}}, \quad \forall t \in \mathbb{R},
$$

where in the last equality we have joined terms with the same exponent. Thus $a_{n}=0$ for all $n$ and, in particular, for the exponent $b_{n_{o}}=\left\langle\alpha_{i_{o} j_{o}}^{k_{o}}+\alpha_{r_{o} s_{o}}^{t_{o}}, \alpha_{o}\right\rangle$, whose coefficient is given by

$$
a_{n_{o}}=c_{i_{o} j_{o}}^{k_{o}} \cdot c_{r_{o} s_{o}}^{t_{o}}\left\langle\pi\left(E_{l m}\right) v_{i_{o} j_{o} k_{o}}, v_{r_{o} s_{o} t_{o}}\right\rangle=0 .
$$

This implies that $c_{i_{o} j_{o}}^{k_{o}} \cdot c_{r_{o} s_{o}}^{t_{o}}=0$, and so part (ii) follows, concluding the proof of the theorem. 


\section{RiCCI FLOW ON LIE GROUPS}

Let $\left(N, g_{0}\right)$ be a (nonnecessarily nilpotent) Lie group endowed with a left-invariant metric with metric Lie algebra $\left(\mathfrak{n},\langle\cdot, \cdot\rangle_{0}\right)$. Let $g(t)$ be a solution to the Ricci flow

$$
\frac{\partial}{\partial t} g(t)=-2 \operatorname{Rc}(g(t)), \quad g(0)=g_{0} .
$$

The short time existence of a solution follows from [Sh89], as $g_{0}$ is homogeneous and hence complete and of bounded curvature. Alternatively, one may require $N$ invariance of $g(t)$ for all $t$, and thus the metric Lie algebra of $(N, g(t))$ would have the form $\left(\mathfrak{n},\langle\cdot, \cdot\rangle_{t}\right)$, where $\langle\cdot, \cdot\rangle_{t}:=g(t)(e)$. The Ricci flow equation (17) is therefore equivalent to the ODE

$$
\frac{\mathrm{d}}{\mathrm{d} t}\langle\cdot, \cdot\rangle_{t}=-2 \operatorname{Rc}\left(\langle\cdot, \cdot\rangle_{t}\right),
$$

where $\operatorname{Rc}\left(\langle\cdot, \cdot\rangle_{t}\right):=\operatorname{Rc}(g(t))(e): \mathfrak{n} \times \mathfrak{n} \longrightarrow \mathbb{R}$, and hence short time existence and uniqueness of the solution in the class of $N$-invariant metrics is guaranteed. In this way, $g(t)$ is homogeneous for all $t$, and hence the uniqueness within the set of complete and with bounded curvature metrics follows from ChZ06. It is actually a simple matter to prove that such a uniqueness result, in turn, implies our assumption of $N$-invariance, as the solution must preserve isometries. The need for this circular argument is due to the fact that the uniqueness of the Ricci flow solution is still an open problem in the noncompact general case (see [Ch09]).

In any case, there is an interval $(a, b) \subset \mathbb{R}$ such that $0 \in(a, b)$ and where existence and uniqueness (within complete and with bounded curvature metrics) of the Ricci flow $g(t)$ starting at $\left(N, g_{0}\right)$ hold.

Remark 5.1. In the case of Lie groups one can use the existence and uniqueness of the solution $g(t)$, forward and backward from any $t \in(a, b)$, to get that the isometry groups satisfy $\mathrm{I}(N, g(t))=\mathrm{I}\left(N, g_{0}\right)$ for all $t$. This fact has recently been proved for the more general class of all complete and with bounded curvature metrics in [K10].

It is easy to prove that if $P(t)$ is the smooth curve of positive definite operators of $\left(\mathfrak{n},\langle\cdot, \cdot\rangle_{0}\right)$ such that

$$
\langle\cdot, \cdot\rangle_{t}=\langle P(t) \cdot, \cdot\rangle_{0},
$$

then the Ricci flow equation (18) determines the following ODE for $P(t)$ :

$$
\frac{\mathrm{d}}{\mathrm{d} t} P(t)=-2 P(t) \text { Ric }_{t}, \quad P(0)=I,
$$

where $\operatorname{Ric}_{t}:=\operatorname{Ric}(g(t))(e): \mathfrak{n} \longrightarrow \mathfrak{n}$ is the Ricci operator.

In this section, we aim to understand under what conditions on the starting metric $\left(N, g_{0}\right)$ one has that the Ricci flow solution $g(t)$ is diagonal, in the sense that $P(t)$ is diagonal when written in some fixed orthonormal basis of $\left(\mathfrak{n},\langle\cdot, \cdot\rangle_{0}\right)$ for all $t \in(a, b)$ (in such a case the metric $g_{0}$ will be called Ricci flow diagonal). It is easy to check that this is equivalent to having the same property for $\operatorname{Ric}_{t}$ (see (19)) and also to the commutativity of the family of symmetric operators $\{P(t): t \in(a, b)\}$.

Clearly, the existence of an orthonormal stably Ricci-diagonal basis $\beta$ for $\left(\mathfrak{n},\langle\cdot, \cdot\rangle_{0}\right)$ implies that $g(t)$ is diagonal, as the diagonal matrices with respect to $\beta$ are invariant under the ODE (19). It follows from Theorem 1.1 that when $N$ is nilpotent, stably Ricci-diagonal bases must necessarily be nice, which shows that this is really too strong a condition to ask of a generic left-invariant metric and, even worse, of any left-invariant metric if the nilpotent Lie algebra $\mathfrak{n}$ happens to have no nice basis whatsoever. 
We now show, as an application of Theorem 1.1, that the existence of an orthonormal stably Ricci-diagonal basis for $\left(\mathfrak{n},\langle\cdot, \cdot\rangle_{0}\right)$ is not necessary to get a diagonal Ricci flow solution $g(t)$.

Example 5.2. $\left(N, g_{0}\right)$ is said to be an algebraic soliton if $\mathrm{Ric}_{0}=c I+D$ for some $c \in \mathbb{R}$ and $D \in \operatorname{Der}(\mathfrak{n})$ (these metrics are called in the literature nilsolitons and solvsolitons in the case when $N$ is nilpotent or solvable, respectively). When $N$ is simply connected, this condition implies that $\left(N, g_{0}\right)$ is indeed a Ricci soliton, as it follows that $\operatorname{Rc}\left(g_{0}\right)=c g_{0}-\frac{1}{2} L_{X_{D}} g_{0}$, where $X_{D}$ denotes the field determined by the one-parameter group of automorphisms of $N$ with derivatives $e^{t D} \in \operatorname{Aut}(\mathfrak{n})$. It is easy to check that in this case,

$$
P(t)=(-2 c t+1) e^{\frac{\log (-2 c t+1)}{c} D}=e^{\frac{\log (-2 c t+1)}{c} \operatorname{Ric}_{0}}
$$

is the solution to (19), and thus the Ricci flow $g(t)$ is always diagonal for algebraic solitons. Indeed, if $\left\{X_{1}, \ldots, X_{n}\right\}$ is an orthonormal basis of eigenvectors of $\mathrm{Ric}_{0}$ with respective eigenvalues $r_{1}, \ldots, r_{n}$, then

$$
P(t)=\operatorname{Diag}\left((-2 c t+1)^{r_{1} / c}, \ldots,(-2 c t+1)^{r_{n} / c}\right),
$$

which generalizes to the class of all algebraic solitons results on the asymptotic behavior of some nilsolitons obtained in [P10, Theorem 2.2] and [W111].

Any nilsoliton is therefore Ricci flow diagonal, though the Lie algebras of many of them do not admit any nice basis and consequently do not admit stably Riccidiagonal bases by Theorem 1.1. The lowest-dimensional example of a nilsoliton whose nilpotent Lie algebra does not admit any nice basis is given in Proposition 2.1 and has dimension 6 . As another example of a nilsoliton which does not admit any nice basis we can take the free 3 -step nilpotent Lie algebra in 3 generators (see N08a $)$.

Remark 5.3. We take this opportunity to point out that the explicit nilsoliton metric for the group in Proposition 2.1 given in [W03, Example 3.4] is wrong. The formula for the Ricci operator of any diagonal metric is not always diagonal as is asserted there. Nevertheless, the existence of a nilsoliton metric (with the same eigenvalue type) can be proved by using an approach similar to [F11, Example 3.3]. Furthermore, the explicit expression for the nilsoliton,

$$
\begin{gathered}
\tilde{\mu}\left(e_{1}, e_{2}\right)=\frac{\sqrt{105}}{42} e_{3}+\frac{\sqrt{70}}{35} e_{4}, \quad \tilde{\mu}\left(e_{1}, e_{3}\right)=\frac{1}{\sqrt{7}} e_{5}, \quad \tilde{\mu}\left(e_{1}, e_{4}\right)=\frac{1}{\sqrt{42}} e_{5}, \\
\tilde{\mu}\left(e_{1}, e_{5}\right)=\frac{1}{\sqrt{10}} e_{6}, \quad \tilde{\mu}\left(e_{2}, e_{4}\right)=\frac{\sqrt{105}}{30} e_{6},
\end{gathered}
$$

was generously provided to us by Fernández Culma, who obtained it by applying the method in [F11, Example 3.2].

In the non-nilpotent case, examples of diagonal Ricci flow solutions which are not written in a nice basis are much easier to find, as the following 3-dimensional solvable example shows.

Example 5.4. Let $\mathfrak{s}_{3}$ be the 3-dimensional Lie algebra defined by

$$
\left[X_{1}, X_{3}\right]=X_{2}+X_{3} .
$$


The basis $\left\{X_{1}, X_{2}, X_{3}\right\}$ is not nice, but it is stably Ricci-diagonal. Indeed, for any inner product $\langle\cdot, \cdot\rangle$ such that

$$
\left\langle X_{i}, X_{i}\right\rangle=a_{i}^{2}, \quad a_{i}>0, \quad\left\langle X_{i}, X_{j}\right\rangle=0 \quad \forall i \neq j,
$$

we use formula (20) from the Appendix to get that $H=\frac{1}{a_{1}^{2}} X_{1}$, and thus for all $r \neq s$ the corresponding Ricci tensor satisfies

$$
\begin{aligned}
\operatorname{Rc}\left(X_{r}, X_{s}\right) & =\frac{1}{4} \sum\left\langle\left[\frac{1}{a_{i}} X_{i}, \frac{1}{a_{j}} X_{j}\right], X_{r}\right\rangle\left\langle\left[\frac{1}{a_{i}} X_{i}, \frac{1}{a_{j}} X_{j}\right], X_{s}\right\rangle \\
& -\frac{1}{2}\left\langle\left[H, X_{r}\right], X_{s}\right\rangle-\frac{1}{2}\left\langle X_{r},\left[H, X_{s}\right]\right\rangle .
\end{aligned}
$$

This clearly vanishes if either $r$ or $s$ is 1 and if

$$
\begin{aligned}
\operatorname{Rc}\left(X_{2}, X_{3}\right) & =\frac{1}{2}\left\langle\left[\frac{1}{a_{1}} X_{1}, \frac{1}{a_{3}} X_{3}\right], X_{2}\right\rangle\left\langle\left[\frac{1}{a_{1}} X_{1}, \frac{1}{a_{3}} X_{3}\right], X_{3}\right\rangle-\frac{1}{2}\left\langle X_{2},\left[\frac{1}{a_{1}^{2}} X_{1}, X_{3}\right]\right\rangle \\
& =\frac{1}{2}\left(\frac{a_{2}}{a_{1}}\right)^{2}-\frac{1}{2}\left(\frac{a_{2}}{a_{1}}\right)^{2}=0 .
\end{aligned}
$$

This example also shows that, beyond nilpotent Lie groups, Theorem 1.1 is no longer true.

On the other hand, in the nonnilpotent case, it is also possible to find nice bases which are not stably Ricci-diagonal, as the following two examples show. In the first one, what fails to be diagonal is the 'unimodularity' part $S(\operatorname{ad} H)$ in formula (20), while in the second one the Killing form part $B$ is not diagonal.

Example 5.5. Let $\mathfrak{s}_{4}$ be the solvable Lie algebra defined by

$$
\left[X_{1}, X_{2}\right]=2 X_{3}, \quad\left[X_{1}, X_{3}\right]=X_{2}, \quad\left[X_{1}, X_{4}\right]=X_{4} .
$$

If $\langle\cdot, \cdot\rangle$ is the inner product on $\mathfrak{s}_{4}$ for which the basis $\left\{X_{1}, \ldots, X_{4}\right\}$ is orthonormal, then the Ricci operator is

$$
\operatorname{Ric}_{\langle\cdot, \cdot\rangle}=\left[\begin{array}{cccc}
-\frac{11}{2} & 0 & 0 & 0 \\
0 & -\frac{3}{2} & -\frac{3}{2} & 0 \\
0 & -\frac{3}{2} & \frac{3}{2} & 0 \\
0 & 0 & 0 & -1
\end{array}\right]
$$

Notice that the basis is however nice.

Example 5.6. Let $\left\{X_{1}, X_{2}, X_{3}\right\}$ be a basis of $\mathfrak{s l}_{2}(\mathbb{R})$ such that

$$
\left[X_{1}, X_{2}\right]=X_{2}, \quad\left[X_{1}, X_{3}\right]=-X_{3}, \quad\left[X_{2}, X_{3}\right]=X_{1} \text {. }
$$

This basis is nice, but the Ricci operator of the metric which makes it orthonormal equals

$$
\operatorname{Ric}=\left[\begin{array}{ccc}
-\frac{3}{2} & 0 & 0 \\
0 & -1 & -1 \\
0 & -1 & -\frac{1}{2}
\end{array}\right]
$$

We finally provide an example of a left-invariant metric on a 4-dimensional nilpotent Lie group whose Ricci flow is not diagonal. Some other left-invariant metrics on this group were proved to be Ricci flow diagonal in [IJL06, Proposition 3.5].

Example 5.7. Let $\mathfrak{n}_{4}$ be the 4-dimensional 3-step nilpotent Lie algebra defined by

$$
\left[X_{1}, X_{2}\right]=\sqrt{2} X_{3}+\sqrt{2} X_{4}, \quad\left[X_{1}, X_{3}\right]=\sqrt{2} X_{4} \text {. }
$$


If $\langle\cdot, \cdot\rangle_{0}$ is the inner product on $\mathfrak{n}_{4}$ for which the basis $\left\{X_{1}, \ldots, X_{4}\right\}$ is orthonormal, then the Ricci operator of the corresponding nilmanifold $\left(N_{4}, g_{0}\right)$, written in this basis, is given by

$$
\operatorname{Ric}_{0}=\left[\begin{array}{cccc}
-3 & 0 & 0 & 0 \\
0 & -2 & -1 & 0 \\
0 & -1 & 0 & 1 \\
0 & 0 & 1 & 2
\end{array}\right]
$$

The following is an orthonormal basis of eigenvectors of $\mathrm{Ric}_{0}$, which is unique up to \pm 1 -scaling and permutations:

$$
\begin{array}{ll}
Y_{1}=(1,0,0,0), & Y_{2}=\left(0,-\frac{1}{2}-\frac{1}{\sqrt{6}},-\frac{1}{\sqrt{6}}, \frac{1}{2}-\frac{1}{\sqrt{6}}\right), \\
Y_{3}=\frac{1}{\sqrt{6}}(0,1,-2,1), & Y_{4}=\left(0,-\frac{1}{2}+\frac{1}{\sqrt{6}}, \frac{1}{\sqrt{6}}, \frac{1}{2}+\frac{1}{\sqrt{6}}\right),
\end{array}
$$

with respective eigenvalues $-3,-\sqrt{6}, 0, \sqrt{6}$. Let us assume that the Ricci flow $g(t)$ starting at $\left(N_{4}, g_{0}\right)$ is diagonal. Thus there exists an orthonormal basis of $\left(\mathfrak{n}_{4},\langle\cdot, \cdot\rangle_{0}\right)$ such that the corresponding matrix of $\operatorname{Ric}_{t}$ is diagonal for all $t$, in particular, for $t=0$, and so such a basis must be $\left\{Y_{1}, \ldots, Y_{4}\right\}$ up to \pm 1 -scaling and permutations. This implies that both $\operatorname{Ric}_{t}$ and the solution $P(t)$ to the ODE (19) are diagonal with respect to the basis $\left\{Y_{1}, \ldots, Y_{4}\right\}$ for all $t$, say $P(t)=\operatorname{Diag}\left(a^{-2}, b^{-2}, c^{-2}, d^{-2}\right)$, $a, b, c, d>0$. A straightforward computation gives that the Ricci curvature therefore satisfies

$$
\begin{aligned}
& \left\langle\operatorname{Ric}_{t} Y_{2}, Y_{3}\right\rangle_{t}=\frac{a^{2}}{(\sqrt{6}+2)^{2}}\left(\frac{4+\sqrt{6}}{24} \frac{d^{2}}{b c}+\frac{12+5 \sqrt{6}}{8} \frac{c}{b}+\frac{12+5 \sqrt{6}}{8} \frac{b}{c}-\frac{76+31 \sqrt{6}}{24} \frac{b c}{d^{2}}\right)=0, \\
& \left\langle\operatorname{Ric}_{t} Y_{2}, Y_{4}\right\rangle_{t}=\frac{a^{2}}{(\sqrt{6}+2)^{2}}\left(\frac{4+\sqrt{6}}{24} \frac{b d}{c^{2}}+\frac{12+5 \sqrt{6}}{8} \frac{d}{b}+\frac{12+5 \sqrt{6}}{8} \frac{b}{d}-\frac{76+31 \sqrt{6}}{24} \frac{c^{2}}{b d}\right)=0, \\
& \left\langle\operatorname{Ric}_{t} Y_{3}, Y_{4}\right\rangle_{t}=\frac{a^{2}(5+2 \sqrt{6})}{12(\sqrt{6}+2)^{2} b^{2} c d}\left(b^{4}-c^{2} d^{2}\right)=0 .
\end{aligned}
$$

All positive solutions to this system are easily seen to satisfy $b^{2}=c^{2}=d^{2}$. This in turn implies that

$$
\left\langle\operatorname{Ric}_{t} Y_{2}, Y_{2}\right\rangle=0, \quad\left\langle\operatorname{Ric}_{t} Y_{3}, Y_{3}\right\rangle=\frac{a^{2}(12+5 \sqrt{6})}{(\sqrt{6}+2)^{2}},
$$

and hence $b^{2}$ and $c^{2}$ cannot solve the ODE system determined by (19) and still be equal to each other, a contradiction. We conclude that the Ricci flow $g(t)$ starting at the nilmanifold $\left(N_{4}, g_{0}\right)$ is not diagonal with respect to any orthonormal basis.

\section{Appendix: Ricci Curvature of left-Invariant metrics}

In this section we give a formula for the Ricci operator of a left-invariant metric on a Lie group with corresponding metric Lie algebra $(\mathfrak{g},\langle\cdot, \cdot\rangle)$. There exists a unique element $H \in \mathfrak{g}$ such that $\langle H, X\rangle=\operatorname{tr} \operatorname{ad} X$ for any $X \in \mathfrak{g}$. If $B$ denotes the symmetric operator defined by the Killing form of $\mathfrak{g}$ relative to $\langle\cdot, \cdot\rangle($ i.e. $\langle B X, Y\rangle=$ $\operatorname{tr}$ ad $X$ ad $Y$ for all $X, Y \in \mathfrak{g})$, then the Ricci operator Ric of $(\mathfrak{g},\langle\cdot, \cdot\rangle)$ is given by (see for instance [B87, 7.38])

$$
\text { Ric }=M-\frac{1}{2} B-S(\operatorname{ad} H),
$$

where $S(\operatorname{ad} H)=\frac{1}{2}\left(\operatorname{ad} H+(\operatorname{ad} H)^{t}\right)$ is the symmetric part of ad $H$ and $M$ is the symmetric operator such that $\langle M X, Y\rangle$ equals the right hand side of formula (1) for all $X, Y \in \mathfrak{g}$, where $\left\{X_{i}\right\}$ can be any orthonormal basis of $(\mathfrak{g},\langle\cdot, \cdot\rangle)$. 


\section{ACKNOWLEDGEMENT}

The authors would like to thank Edison Fernández Culma for very helpful comments on a first version of this paper.

\section{REFERENCES}

[B87] A. Besse, Einstein manifolds, Ergeb. Math. 10 (1987), Springer-Verlag, BerlinHeidelberg. MR867684 (88f:53087)

[CS09] X. CAO, L. SALOFF-COSTE, Backward Ricci flow on locally homogeneous three-manifolds, Comm. Anal. Geom. 17 (2009), 305-325. MR2520911(2010k:53095)

[C96] R. CARLES, Weight systems for complex nilpotent Lie algebras and application to the varieties of Lie algebras. Publ. Univ. Poitiers, 96 (1996)

[Ch09] B.-L. Chen, Strong uniqueness of the Ricci flow, J. Diff. Geom. 82 (2009), 336-382. MR:2520796 (2010h:53095)

[ChZ06] B.-L. Chen, X.-P. Zhu, Uniqueness of the Ricci flow on complete noncompact Riemannian manifolds, J. Diff. Geom. 74 (2006), 119-154. MR2260930 (2007i:53071)

[F11] E. Fernandez Culma, Classification of 7-dimensional Einstein nilradicals, Transform. Groups 17 (2012), 639-656. MR2956161

[dG07] W. A. DE GraAf, Classification of 6-dimensional nilpotent Lie algebras over fields of characteristic not 2, J. Algebra 309 (2007), 640-653. MR.2303198 (2007k:17012)

[G08] D. Glickenstein, Riemannian groupoids and solitons for three-dimensional homogenous Ricci and cross-curvature flows, Int. Math. Res. Not. 12 (2008). MR2426751 (2009f:53100)

[GP10] D. Glickenstein, T. Payne, Ricci flow on three-dimensional, unimodular metric Lie algebras, Comm. Anal. Geom. 18 (2010), 927-962. MR.2805148

[IJ92] J. IsEnBERG, M. JACKSON, Ricci flow of locally homogeneous geometries on closed manifolds, J. Diff. Geom. 35 (1992), 723-741. MR1163457 (93c:58049)

[IJL06] J. Isenberg, M. Jackson, P. Lu, Ricci flow on locally homogeneous closed 4-manifolds, Comm. Anal. Geom. 14 (2006), 345-386. MR2255014(2007e:53087)

[KM01] D. Knopf, K. MCLeOD, Quasi-convergence of model geometries under the Ricci flow, Comm. Anal. Geom. 9 (2001), 879-919. MR.1868923(2003j:53106)

[K10] B.L. Kotschwar, Backwards uniqueness for the Ricci flow, Int. Math. Res. Not. 2010 (2010), no. 21, 4064-4097. MR2738351(2012c:53100)

[L06] J. LAURET, A canonical compatible metric for geometric structures on nilmanifolds, Ann. Global Anal. Geom. 30 (2006), 107-138. MR2234091(2007g:53092)

[L09] , Einstein solvmanifolds and nilsolitons, Contemp. Math. 491 (2009), 1-35. MR2537049 (2010g:53081)

[L10] The Ricci flow for simply connected nilmanifolds, Comm. Anal. Geom. 19 (2011), 831-854. MR2886709

[LW11] J. LAURET, C.E. WILL, Einstein solvmanifolds: existence and non-existence questions, Math. Annalen 350 (2011), 199-225. MR2785768

[Lt07] J. LotT, On the long-time behavior of type-III Ricci flow solutions, Math. Ann. 339 (2007), 627-666. MR2336062 (2008i:53093)

[Mg07] L. MAGnin, Adjoint and trivial cohomology tables for indecomposable nilpotent Lie algebras of dimension $\leq 7$ over $\mathbb{C}$, e-Book, 2nd Corrected Edition, 2007.

[M76] J. Milnor, Curvature of left-invariant metrics on Lie groups, Adv. Math. 21 (1976), 293-329. MR0425012 (54:12970)

[N08a] Y. NikolayeVsky, Einstein solvmanifolds with free nilradical, Ann. Global Anal. Geom. 33 (2008), 71-87. MR2369187(2008m:53120)

[N08b] _ Einstein solvmanifolds with a simple Einstein derivation, Geom. Ded. 135 (2008), 87-102. MR2413331(2009f:53064)

[N11] Einstein solvmanifolds and the pre-Einstein derivation, Trans. Amer. Math. Soc. 363 (2011), 3935-3958. MR2792974 (2012f:53095)

[P10] T. PAYne, The Ricci flow for nilmanifolds, J. Modern Dyn. 4 (2010), 65-90. MR.2643888 (2011d:53163)

[S01] S. Salamon, Complex structures on nilpotent Lie algebras, J. Pure Appl. Algebra 157 (2001), 311-333. MR:1812058 (2002g:53089) 
[Sh89] W.X. SHI, Deforming the metric on complete Riemannian manifolds, J. Diff. Geom. 30 (1989), 223-301. MR:1001277 (90i:58202)

[W03] C.E. Will, Rank-one Einstein solvmanifolds of dimension 7, Diff. Geom. Appl. 19 (2003), 307-318. MR2013098 (2004j:53060)

[W11] The space of solvsolitons in low dimensions, Ann. Global Anal. Geom. 40 (2011), 291-309. MR2831460 (2012j:53053)

[W111] M.B. Williams, Explicit Ricci solitons on nilpotent Lie groups, J. Geom. Anal. 23 (2013), 47-72. MR3010272

[Wi82] E. Wilson, Isometry groups on homogeneous nilmanifolds, Geom. Ded. 12 (1982), 337346. MR661539 (84a:53048)

FamaF and Ciem, Universidad Nacional de Córdoba, Córdoba, Argentina

E-mail address: lauret@famaf.unc.edu.ar

FamaF and Ciem, Universidad Nacional de Córdoba, Córdoba, Argentina

E-mail address: cwill@famaf.unc.edu.ar 